\title{
HP-Bio: High Pressure BioSAXS for Deep Life and Extreme Biophysics
}

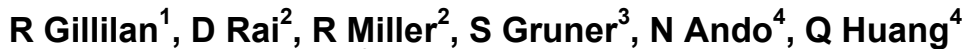 \\ ${ }^{1}$ MacCHESS, Cornell Univ, Ithaca, NY, ${ }^{2}$ Cornell High Energy Synchrotron Source, Ithaca, NY, \\ ${ }^{3}$ Physics Dept. \& CHESS, Cornell Univ, ${ }^{4}$ Cornell University, Ithaca, NY \\ reg8@cornell.edu
}

The structural biology and biophysics of biomolecules under extreme pressure have attracted an increased interest in recent years with the discovery of copious microbial life in extreme environments such as rock pores kilometers down in the deep hot subsurface, cold ocean trenches $11 \mathrm{~km}$ deep, and hydrothermal vents as hot as $122^{\circ} \mathrm{C}$. How proteins and other biomolecules are able to function and maintain integrity under such extreme conditions that would normally destroy surface life is a question of practical importance for food and pharmaceutical preparation. It may also shed light on the origins and limits of life, as well as the potential for life elsewhere in the universe. Biophysically speaking, virtually anything a biomolecule does changes its volume and hence is potentially influenced by pressure. Consequently, pressure offers a unique way to probe cavities and volume changes in biological systems, and to unfold or dissociate systems in a controlled way. The Cornell High Energy Synchrotron Source now hosts a facility dedicated to high-pressure structural biology. Two of our main technologies will be discussed here: "batch" high pressure BioSAXS that can reach pressures in excess of $400 \mathrm{MPa}$, and a novel highpressure chromatography-coupled BioSAXS system that can simultaneously separate and measure biomolecules at pressures of up to $100 \mathrm{MPa}(\sim 10 \mathrm{~km}$ deep in the ocean). This talk will cover design, performance, practical considerations for doing experiments, and biological applications.

Acta Cryst. (2020). A76, a136 